\title{
Proximal Femoral Allograft in Two-Stage Revision for Failed Septic Hip Arthroplasty
}

\author{
Alidou Traore1, Karim Tribak², Jeancis Be ${ }^{1}$, Maité Van Cauter², Christelle Mobiot-Aka1, \\ Yaya Sidi Traoré1, Slim Alban Mbende ${ }^{3}$, Daouda Kanaté Soumaro', Rebecca Eva Boka1, \\ Jean Cyr Yombi ${ }^{2}$, Christian Delloye², Olivier Cornu² \\ ${ }^{1}$ Trauma and Orthopaedic Unit, Yopougon Teaching Hospital, Abidjan, Cote d'Ivoire \\ ${ }^{2}$ St-Luc University Clinics (UCL), Brussels, Belgium \\ ${ }^{3}$ Neurosurgery Unit, Yopougon Teaching Hospital, Abidjan, Cote d'Ivoire \\ Email: tralidou@yahoo.fr
}

Received 29 October 2015; accepted 15 December 2015; published 18 December 2015

Copyright (C) 2015 by authors and Scientific Research Publishing Inc.

This work is licensed under the Creative Commons Attribution International License (CC BY).

http://creativecommons.org/licenses/by/4.0/

(c) (i) Open Access

\begin{abstract}
Infection after hip prostheses is a potentially devastating complication, and a serious medical and surgical challenge, especially when associated with Paprosky type III femoral bone loosening. Treatment is difficult and options are limited. We report on a 2-stage revision of 15 patients undergoing femur reconstruction with massive allografts. Materials and methods: This was a prospective study which included 15 patients (10 men and 5 women) with infected hip prosthesis, associated with Paprosky type III femoral proximal massive bone loss. The median age of patients was 64 years with a preoperative functional status score of 6 . The average number of procedures to the same hip after the first arthroplasty was 6 . All patients benefited from a 2 -step surgery with massive allografts and locking prosthesis. The average follow-up time was 36 months. Results: Infection was monomicrobial in 14 cases; and was polymicrobial in 4 cases. Methicillin-resistant Staphylococcus epidermidis was the main bacteria $(n=10)$. The average $C$-reactive protein level before the second procedure was $2.3 \pm 3$.4. It was normalized in 8 cases. We recorded 13 cases of primary consolidation without another surgery, 3 cases of relapse, 2 traumatic dislocations and 2 fractures of the allograft. Conclusion: Hip prosthesis infection is a potentially catastrophic complication with significant negative ramifications for both the patient and the healthcare system. Massive allografts use in Paprosky III femoral defect remains very attractive for bone stock restoration and hip function improvement.
\end{abstract}

\section{Keywords}

Arthroplasty, Hip, Infection, Femur, Paprosky 3, Massive Allograft 


\section{Introduction}

Septic loosening poses, besides the major issue of infection, a bone stock restoration problem. Despite an increase in procedures and primary prevention measures, $0.5 \%$ to $2 \%$ of primary arthroplasties will be infected. This rate is higher during revision surgery [1] [2]. Infection is a potentially catastrophic and serious complication both individually and in terms of public health. There is a wide agreement in the literature concerning the cost to both the economy and individuals affected by infection. The cost to the US Social Security is estimated at approximately $\$ 280$ million per year. In Great Britain, the management of infection is estimated at 20,500 50,000 euros per patient [1]-[4].

Clinical and laboratory signs are often nonspecific and inconsequential, making the diagnosis difficult. It is challenging to offer a single attitude vis-à-vis joint prosthesis infection because there is a great deal of factors that enter into the healing process [3]-[6]. Single-step surgery is less aggressive, but has limited indication [4] [7]. Most authors agree on a 2-stage surgery [8]-[10].

Massive femoral allografts are usually executed in oncology surgery and in aseptic revisions of the hip with large femoral bone defect [11] [12]. There is little evidence in the literature supporting their use in septic revisions of the hip with femoral bone resorption [9] [13] [14]. Nonetheless, they remain the best choice for proximal femur reconstruction. Massive femoral allografts executed in septic revision of the hip and in oncology surgery for proximal hip reconstruction have the advantage to fill the bone loss, to allow the insertion of gluteus muscles and provide immediate mechanical reinforcement [11] [12]. In addition to their mechanical and functional aspects, they also allow the release of antibiotics [15].

We report on 15 cases of femoral reconstruction with massive allografts after a 2-stage revision, following septic hip arthroplasty with proximal femur major bone loss.

\section{Material and Methods}

This was a prospective study from 1999 to December 2011 which included 17 patients with infected hip prostheses, associated with Paprosky III femoral bone loss (Figure 1). This study was conducted in University hospital with 4300 orthopaedic surgical procedures a year. All patients benefited from a 2-step surgery; and two were excluded from the study because of a short follow-up period.

Eighteen procedures were performed (2-stages procedure including relapses) on 10 men and 5 women. The median age of patients was 64 years, ranging from 46 to 75, predominantly male, with a striking ratio of 2:1. The average number of procedures to the same hip after the first arthroplasty was 6 with ranges of 2 to 8 . PostelMerle D’Aubigné [16] mean preoperative score was 5 with ranges of 2 to 8.

Clinical features included chronic pains and fistulas in 3 patients. Out of 15 patients assessed preoperatively, 11 diagnoses were confirmed using bacteriological or microbiological tests of joint products after scan-guided puncture. Where doubts arose; prosthetic loosening images on X-ray, CT scan, or even bone scintigraphy or white blood cells uptake images, guided the first step of our treatment.

Risk factors were investigated in all patients, namely: history of arthroplasty revision on the same hip, diabetes, inflammatory diseases (rheumatoid arthritis, lupus), immunosuppressive therapy, cancer and UTI.

Routine blood test included full blood count and CRP. CRP was not only a diagnostic presumption marker, but above all, an infection monitoring and antibiotics effectiveness marker.

\subsection{Treatment}

The surgical approach was guided by previous approaches. The approach was posterior in almost all cases, extending over the femur with or without femorotomy. An additional ilioinguinal approach was executed in one patient due to a pelvic brim loosening with an acetabular implant protrusion.

First stage:

This first step was that of debridement and constituted a treatment milestone. It involved removing all foreign bodies (femoral stem, acetabular implant, whole or broken screws and, if possible, sutures) and excising any infectious or inflammatory tissues around the prosthesis. During bone resection, much of the thin cortical bone must be preserved to allow a double-barrel technique in secondary reconstruction.

At least 6 samples were taken during this stage for bacteriological, pathological and genetic testing. Preoperative positive cultures in those samples were evidence of infection in all patients. An articulated cement spacer was introduced and allowed rapid mobilization of patients and kinesitherapy sessions. 


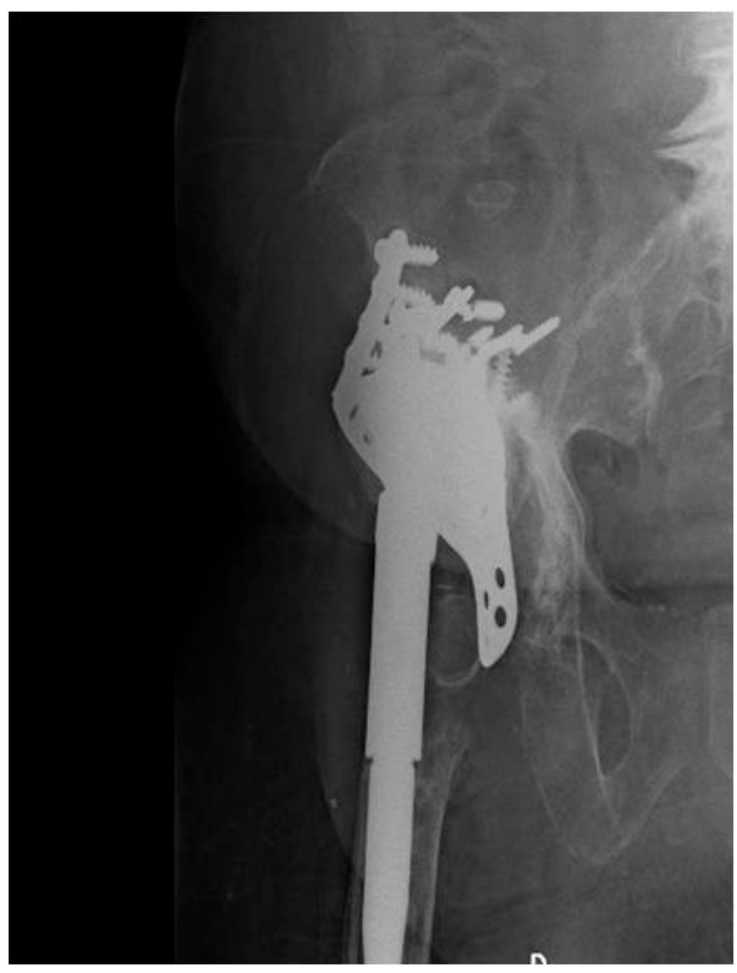

Figure 1. Anteroposterior X-ray of the showing hip loosening prosthesis and massive bone loss (Paprosky 3) of the proximal femur.

Two patients required 2 local flaps and a free one to cover their trochanteric areas. All patients received a 4 week-course of intravenous antibiotics; and were admitted to our unit pending their second surgery, or were referred to a physical rehabilitation hospital after 7 - 10 days of hospitalisation.

Second stage: Prosthetic reconstruction

\section{Implant:}

It is a hydroxyapatite-coated, cement-free locking prosthesis with modular system enabling metaphyseal filling, and diaphyseal length, diameter and curvature adjustment. The prostheses are anatomically curved with a neck-shaft angle of $135^{\circ}$.

\section{Allograft:}

It is a well-planned step, beginning with the selection of the femoral allograft. This selection depends on the patient's femoral X-ray compared to the fluoroscopic images of femoral allografts in the tissue bank. The length of the allograft will depend on the defect to be filled and the shortening to make up. It is then soaked in a solution of rifampicin. Often, femoral condyles of the allograft have been used for the reconstruction of the acetabulum when it has a major bone loss. Diaphyseal medullary canal of the allograft is reamed larger than the chosen stem diameter of the prosthesis.

The proximal end is rasped in such a way that the prosthesis metaphysis lies ideally at Merkel level (Figure 2).

During surgery, bacteriological samples are taken. Surgery is followed by removal of the cement spacer. Whenever possible, we favoured the "double barrel" technique for reconstruction.

Allograft is a dead bone, therefore incapable of inducing a bony-integration with hydroxyapatite. It was cemented on the outside and a glove finger was pushed through locking holes of the stem of the prostheses. The prosthesis was then buried inside the cemented allograft. At this stage, we would proceed to the implantation and locking of the assembled allograft-prosthesis in the remaining diaphyseal medullary canal of patients.

In the double barrel technique, suturing reinforced the allograft to the cortical portion of the femur shaft (Figure 3). In case of a vertical cut of the distal diaphysis of the femur, spongy bone associated with DBM (Demineralized Bone Matrix) was placed at the junction allograft-distal femur to promote consolidation. 


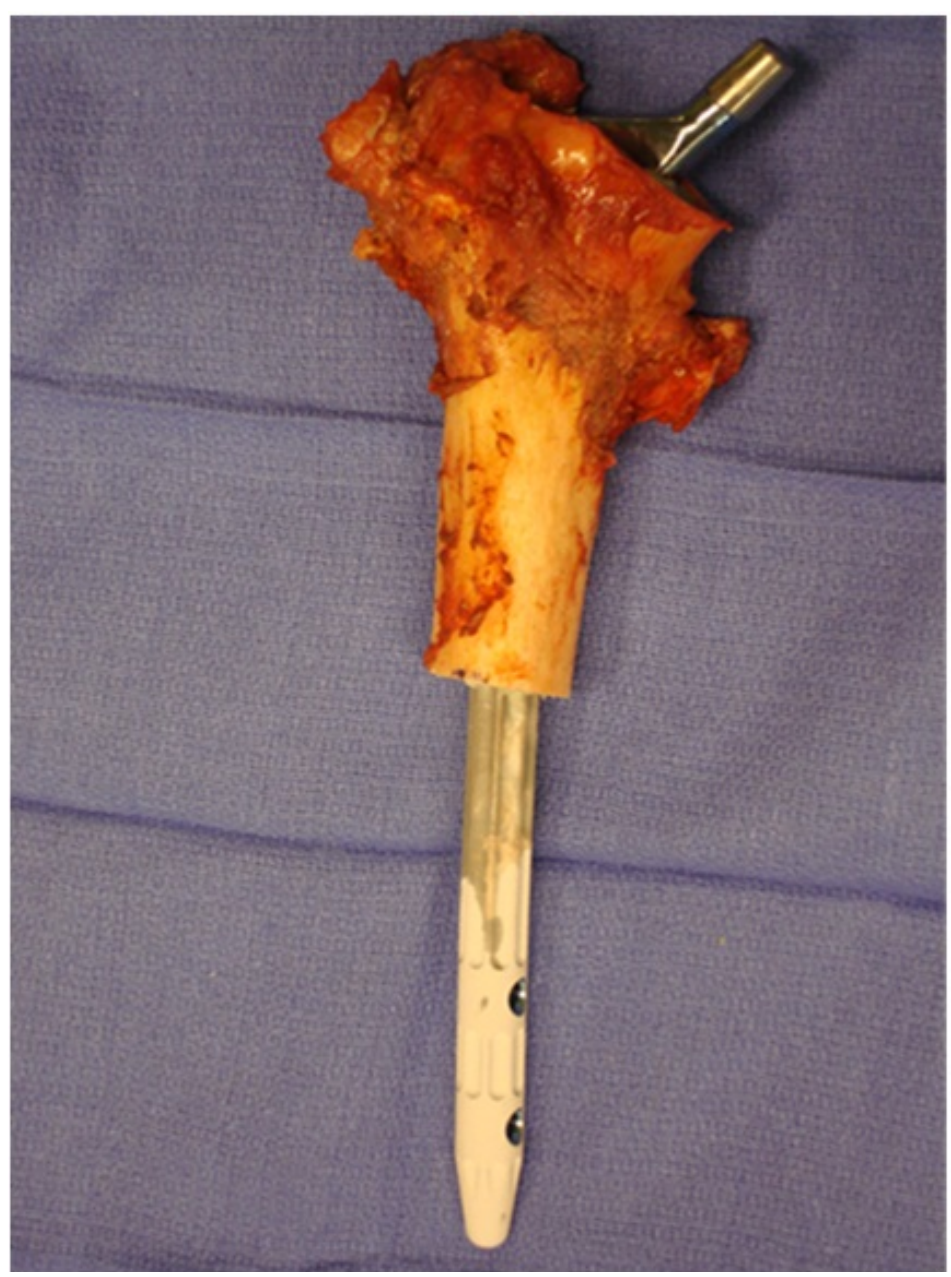

Figure 2. Technique of the introduction of the prosthesis into allograft. The prosthesis metaphysis lies ideally at Merkel level.

Reconstruction of the acetabulum was executed at the same time with a femoral condyle allograft $(n=5)$, an irradiated femoral head $(n=6)$ or lyophilized $(n=5)$. The Kerboul ring was used 15 times with a dual mobility in 13 cases.

Average lengths of allograft and prosthesis were $22 \mathrm{~cm}$ (range of 13 to $31 \mathrm{~cm}$ ) and $31 \mathrm{~cm}$ (range of 26 to 36 $\mathrm{cm}$ ) respectively, and the diameter of the prosthesis averaged $14 \mathrm{~cm}$ (ranging from 13 to $16 \mathrm{~cm}$ ).

Patients received another one week of intravenous antibiotics and continued with tablets for a further 3 months.

Control CRP tests were done every week for 3 months, and then, every 2 weeks from the $3^{\text {rd }}$ to the $6^{\text {th }}$ month, and once a month from the $6^{\text {th }}$ to the $12^{\text {th }}$ month. CRP tests were then checked every semester for 2 years, and normal CRP levels were strong evidence to declare a patient free from infection.

Control x-rays performed at 3, 6, 12 and 24 months, assessed the quality of the modular prostheses-allograft system and callous formation at the junction bone-allograft, and revealed potential mechanical complications.

Average follow-up time was 36 months, and hip function was assessed before and after reconstruction using Postel Merle d'Aubigné hip score.

Statistical analyzes were performed by Chi 2 test $(\mathrm{p}<0.05)$.

\subsection{Ethical Consideration}

Informed consent was obtained from a patient. Agreement was obtained from the local ethical committee of the institution (registration number NCT02355301; 2015/26JAN/025). 


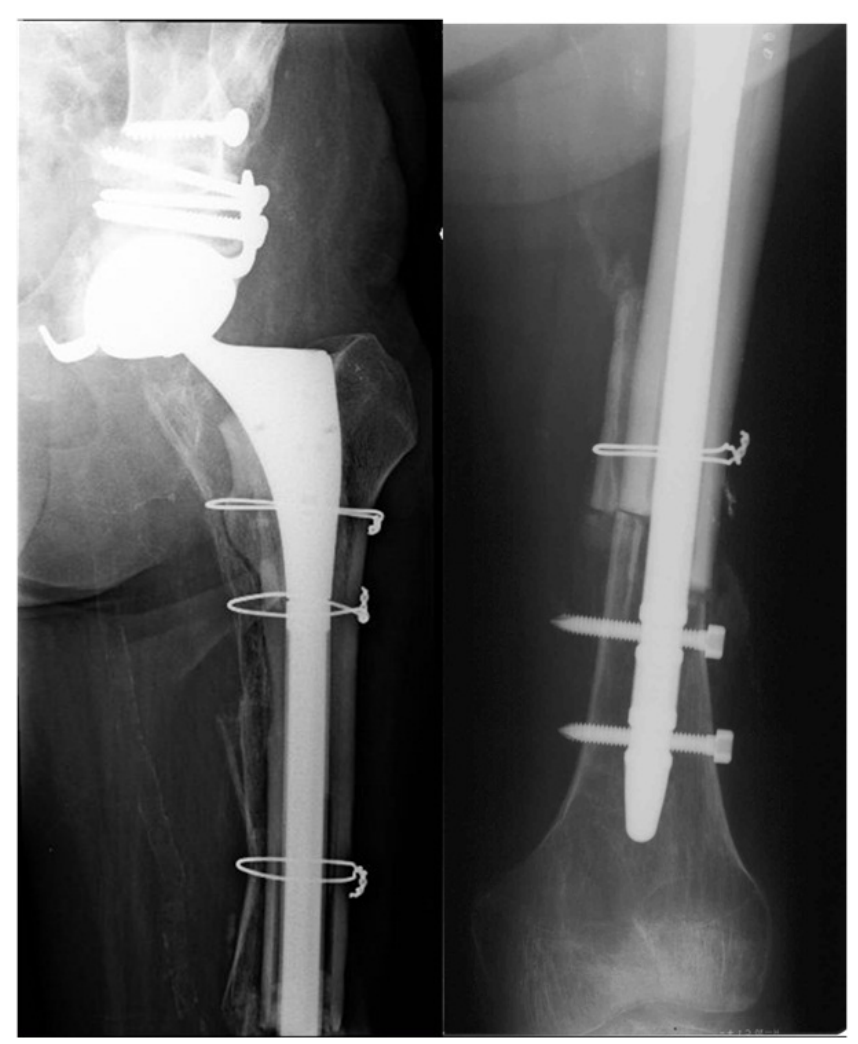

Figure 3. Anteroposterior X-ray of the pelvis and distal femur of two reconstructions with the double barrel technique (A) and step cut technique allograft-host bone (B).

\section{Results}

Average follow-up time was 36 months.

\subsection{Microbiology}

Infection was monomicrobial in 14 cases; and polymicrobial in 4 cases. Methicillin-resistant Staphylococcus epidermidis was the main bacteria $(\mathrm{n}=10)$. The average CRP level before prosthetic reconstruction was $2.3 \pm$ 3.4 and normalised in 8 cases $(<1 \mathrm{mg} / \mathrm{l})$. Nonetheless, surgery was not postponed in the remaining cases; where CRP values were higher.

\subsection{Radiological Results}

We recorded 13 cases of primary consolidation without requiring further procedures (Figure 4).

\subsection{Functional Results}

Postel-Merle d'Aubigné [16] average functional score was 14 with a range of 12 to 16.

\subsection{Complications}

\section{Relapses}

We recorded 3 cases of recurrences at 2, 7 and 20 months. There was no statistical link between relapses and CRP values before reconstruction. Of all 3 recurrences, 2 had normal CRP values before reconstruction. There was no correlation between the positive value of the pre-operative CRP and the occurrence of infection.

Two were caused by MRSE, oxacillin-sensitive Staphylococcus aureus and gram negative bacteria. Revision surgeries were performed successfully at 19, 39 and 52 months respectively. 


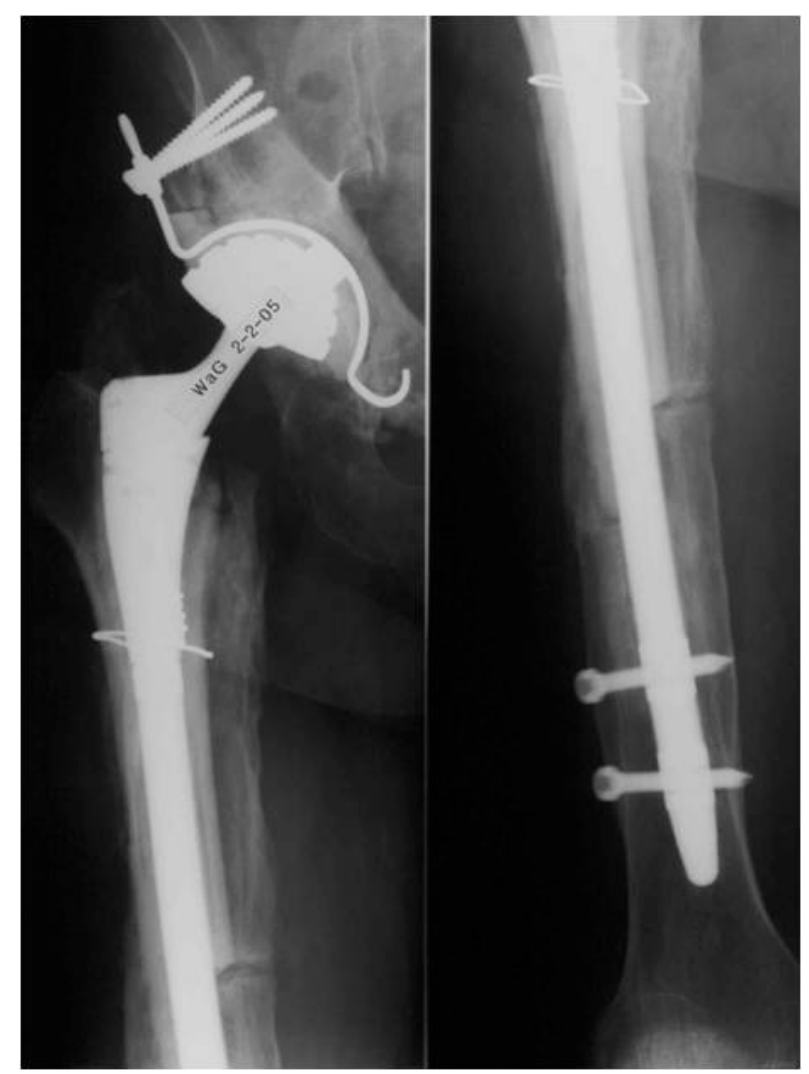

Figure 4. Anteroposterior X-ray of the hip and distal femur 1-year postoperative show allograft-host bone union and absence of allograft resorption.

Out of 18 surgical procedures, 3 were unsuccessful with an infection control success rate of 83.33\%.

\section{Mechanical complications}

We recorded 2 cases of traumatic dislocations and 2 cases of allograft fractures (at 80 and 109 months). Both dislocations were reduced orthopedically. Fractures occurred behind an infection-free background; and were operated after a one-step revision surgery with new allografts which consolidated successfully.

Three broken locking screw were found; in 3 cases they did not impact on the stability of the system, and in one case they were replaced (Figure 5).

\section{The limitations of this study}

Cases study (no control group) and limited number of patients (but such a catastrofic event as an infection of a prosthetic loosen implant with bony destruction is fortunately rare);

Mean follow up lower than 5 years (meaning that some adverse event such as the allograft fracture might further occurred as in the two patients with longer follow up).

\section{Discussion}

Prosthetic infection is a serious complication both individually and in terms of public health [1] [3] [15]. Infection rate in primary arthroplasty is estimated at $1 \%$ to $2 \%$. There is a 10 to 20 fold risk increase in prosthetic revision, reaching $10 \%$ to $40 \%$ by the third revision. Infection should be identified urgently to guide one's therapeutic strategy. This is, however, challenging; since no test has been proved so far to be specifically and sensitively discriminative [3] [5] [15]. Symptoms may be minor and clinically inconsequential, marked by chronic pains and local inflammatory signs. If an infection can be diagnosed on the sole basis of chronic pains after arthroplasty, then the burning question is "which discriminative test is required to confirm or rule out this diagnosis?” Ideally, the diagnosis is straightforward when there is a productive fistula [2] [4] [5]; but, this is an inconstant clinical sign. Out of 15 patients in our series, only 3 were found to have developed productive fistula. 


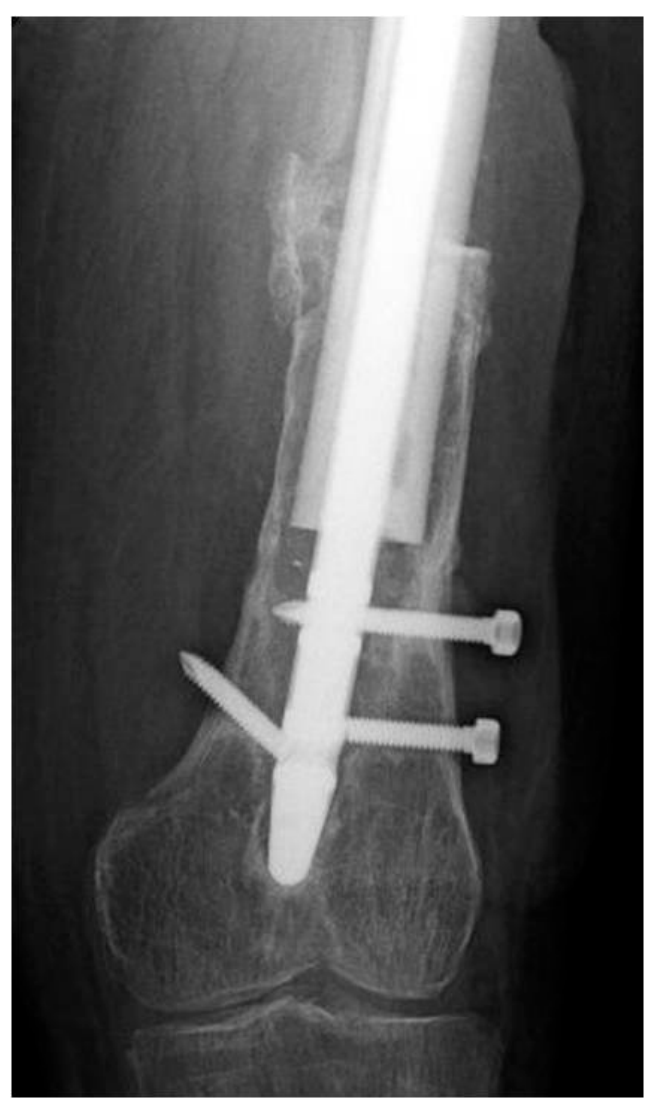

Figure 5. X-ray distal femur showing a breakage of a screw and a migration of the other. These two screws have been replaced.

Routine tests, such as erythrocyte sedimentation rate (ESR) and CRP play an essential discriminative role. Studies have shown that, the two tests combined together constitute an excellent screening tool to rule out an infection [2] [4] [5] [15]; and a CRP above $10 \mathrm{mg} / \mathrm{l}$ combined with an accelerated ESR, have a predictive value of infection in more than 92\%. But CRP values must be interpreted in line with the patient's history of inflammatory processes such as rheumatoid arthritis, neoplastic processes and recent surgeries other than the hip [3] [4] [15] [17] [18]. Fistulas have been associated with abnormally low CRP levels.

A CRP test was performed to all our patients, and constituted not only a presumptive infection marker, but also an antibiotics monitoring and effectiveness marker.

Arthrography puncture of the hip is commonly performed to confirm a clinical suspicion of prosthetic infection. Puncture-aspiration of the hip, when feasible, assists in the identification of bacteria, tests their sensitivity and may guide the choice of surgery. Due to high risk of failures, a one-step technique is not recommended in the presence of certain bacteria such as MRSE, MRSA and Pseudomonas aeruginosa [7]-[9]. In addition, when no bacterium is isolated, leukocytes testing from the joint fluid are warranted and levels greater than 1700 cells/ml have $94 \%$ sensitivity and an $88 \%$ specificity of joint infection [2].

Puncture-aspiration of the hip has its limits. Quite often, there is no fluid coming out; or the puncture is technically not feasible because of peri-prosthetic ossifications, or acetabular implant loosening and moving intra-pelvic as was the case with 3 of our patients.

In any case, patients' history of prosthetic infection as was seen in 12 of our patients; or an infection in other surgical procedures with elements of bone scintigraphy, white blood cells scintigraphy, scanner or standard X-ray signs of septic loosening, guided our first surgical procedure [19] [20].

Bacteriological or genetic confirmation of infection was possible in all patients. In most cases, prosthetic infection was diagnosed before and during surgery [3]-[6].

The treatment goal is to clear the infectious process and restore joint function. The classic scenario is surgery 
with removal or change of the prosthesis in chronic infections. Debridement and retention of the prosthesis [7] or two-stage reimplantation [8]-[10] procedures remain controversial.

Debridement and retention of the prosthesis has the advantage to offer short hospital stay and an overall low cost. Relapses are common, ranging from $25 \%$ to $40 \%$ [3] [4]. When microbiological testing fails to isolate the bacteria before surgery, a debridement and retention of the prosthesis procedure offers no alternative, rather than commencing empirical antibiotics postoperatively with the prosthesis in place. A second drawback of this technique is the difficulty in determining infection-free areas during surgery, leaving the surgeon with no option than to perform an extensive debridement [4] [7]. Single-stage treatment, especially a prosthetic salvage procedure, must comply with major criteria of short timing between symptoms and debridement (on average 2 - 14 days), and minor criteria such as fistula, radiological signs of loosening and a graft-free indication. In addition, it must take into account the type and virulence of the bacteria [4] [7].

Most authors agree that the 2-stage surgery is the technique of choice, especially in revision surgery and in chronic infections [8]-[10].

All our patients benefited from this technique. Critics of this procedure deplore its impact on patients' quality of life due to prolonged hospital stay between surgeries. The introduction of manufactured or custom-made temporary spacer composed of proximal femur prosthesis wrapped with cement and antibiotics, reduced patients' hospital stay and improved morbidity significantly.

The spacer provided stability to the hip. Early postoperative mobilization and wandering without weightbearing were allowed. Moreover, second-stage procedures were made easy thanks to good spacer exposure. Our study recorded a 16-hour/day skeletal traction time and pain-free days from the $5^{\text {th }}$ and the $6^{\text {th }}$ day. The average waiting time between procedures was 4 weeks; however, longer waiting times were recorded in 3 cases, in which a 3rd debridement $(n=2)$ or a 4th debridement $(n=1)$ was warranted on the grounds of persistent high CRP levels, or isolation of bacteria after bacteriological testing of drainage tubes. Local signs and CRP levels were important indicators for the second-stage treatment. The majority of CRP levels normalized between the $3^{\text {rd }}$ and the $4^{\text {th }}$ week, except in cases of hematoma or bruising of the spacer. We recorded contamination of intravenous or urinary routes, which disrupted biological testing and increased CRP levels; but was not related to a persistent infectious process. This, with other arguments, justified our choice of a four-week antibiotics protocol rather than a six-week one.

Second-stage procedures were performed in some patients with CRP levels around normal values (between 1 to $3 \mathrm{mg} / \mathrm{l}$ ), normal values being less than 1 . There was no statistical significance between relapse and CRP levels before reconstruction.

Antibiotics follow surgery and constitute the treatment backbone, but should be given after bacteriological testing has been conclusive. To date, there is no codified protocol; and an ideal protocol and its timing still raise a great deal of controversies. Conventionally, it is a 3-month protocol for a single-stage surgery [4] [7] and a 4 to12-week protocol for a double-step procedure [3] [5] [6]. There are, however, exceptions related to patient comorbidities, the surgical technique and the causative bacteria. Antibiotics must be chosen on the merits of their sensitivity and patient tolerance. Unfortunately and occasionally, patients' economic situation or social factors (insurance, social welfare) have hindered the choice of antibiotics. Generally speaking, intravenous antibiotics are administered for 4 weeks between the first and the second procedure, and a week after recons-truction, followed by three months of tablets.

Staphylococcal infections were treated with antibiotics with proven good tissue penetration, such as Rifampicin, combined with a fluoroquinolone. A weekly control CRP test for 3 months, then monthly control, 6-monthly and annually was the single infection monitoring marker set in the study. Normal CRP levels for a 2-year period were strong evidence to declare a patient free from infection. Liver functions were also monitored throughout the rifampicin treatment [15].

Revision of the infected hip arthroplasty with major bone loss is difficult, options are limited. Treatment options vary and include palliative treatment consisting of prosthesis resection (pseudo-resection of the head and neck) [12]. This technique is, however, unacceptable because of dismal functional outcome. Arthrodesis is technically challenging due to the extent of defect [21].

Reconstruction techniques are all innovative and include the use of synthetic bone substitutes of cortical allograft, cancellous bone impaction, with varying fixation techniques [9] [10] [12]-[14] [22]-[26]. Most of these techniques are, however, limited to minor bone loss classified as Paprosky I and II, and to a lesser extent as Paprosky III. 
Massive allografts are common indications in oncologic surgery and aseptic hip prostheses revision [11] [12] [21]. There is little in the literature to support their indication in septic revisions [9] [13] [14]. Their use is, however, attractive; because they restore bone stock and provide immediate mechanical reinforcement in contrast to metal implants such as mega or custom-made prostheses. As a result, they reduce significantly the risk of instability. Moreover, the allograft has an inherent ability to bind with the distal femur and become an integral part of the femur, to restore the limb anatomy, and to release antibiotics as it is wrapped with Rifampicin before implantation [15]. From one centre to another, indications vary; but, in Paprosky III as in all our patients, allografts are often indicated.

Our $83.33 \%$ success rate is hardly comparable to other series in the literature. The two most quoted studies in the literature were reported by Alexeeff [13] and Nusem [9]. Nusem [9] reported a sample of 10 patients, in which 6 were classified as Paprosky I and 4 as Paprosky III. Only one patient who underwent prosthetic resection as a mean of controlling infection had a recurrence. Alexeeff [13] reported a series of 11 massive allografts performed in 17 patients, and found no relapse. Halliday [27] found 7.54\% of recurrences in impaction techniques; other studies with heterogeneous groups such as fragmented allograft, allograft cortical rods, found recurrence rates of $9 \%$ and $14 \%$ in first or second revision [10] [13] [14].

The risk of recurrence might be associated to many factors, mainly incomplete debridement with remains of cement or foreign bodies, the virulence of bacteria, positive cultures during second-revision, history of infection on the same hip and factors related to the patient [28]-[31].

Rigid implantation of the allograft has unknown consequences. Methods of allograft fixation include cementation into the distal femur [11], the use of long prosthesis with or without distal locking or step-cut technique combined with pins or plates fixation [9] [13]. Biomechanical studies conducted on cadavers demonstrate a good stability of distal cementation systems [32]-[34]. There is, however, a high risk of non-union. Distal femur cementation prevents further reconstructions, should there be a serious indication of another reconstruction.

We favoured "Amplitude" Ortho hip prosthesis, which allowed early mobilization and revision without damaging distal femur bone stock. During revisions for the 3 recurrences and the 2 allograft fractures, we were able to assemble the same device using equivalent or longer femoral stems for bridging locking holes in order to reduce the risk of distal femur fractures on locking holes.

We recorded good or excellent functional outcome in our series of 15 patients compared with functional scores on admission. But, there was no statistical link between the functional score and the length of bone resection. This excellent outcome could also be explained by the use of a spacer, which allowed wandering and physical rehabilitation sessions. Unlike Nusem [9] and Alexeeff [13] series, we didn't perform any pseudo-resection for infection control purposes. That score remained good, with good distal femur-allograft consolidation, even in cases where relapses occurred. Only one patient developed a productive fistula.

The uses of massive allografts remains attractive, however, there are still limiting factors, such as the necessity of a reliable tissue bank with quality allografts in sufficient number. Infection rates in oncologic surgery vary from $0 \%$ to $19 \%$ [9]-[14]. There is still a great deal of controversy on the explanation of occurrence of these infections after allograft surgery, because of possible involvement of multifactorial factors such as immunosuppressive treatments, complexity and timing of procedures, extensive soft tissue dissection and comorbidities [15] [28] [31].

Other complications such as nonunion and fractures are long-term complications. We did not record any case of nonunion in our series. The main factors underlying this phenomenon may be our double-barrel technique, and the extent of DBM interdigitation at the junction distal femur-allograft [25]. We have, however, recorded two cases of fractures. Causes of massive allograft fractures are still unclear. Generally speaking, when they occur before 12 months, loosening due to screw holes is the main factor. After 12 months they are constraint fractures, after 24 months they are stress related, and beyond 48 months, they are due to massive allograft resorption [35].

In two cases where fractures occurred behind an infection free background, single-stage revisions with new allograft were performed successfully.

\section{Interests:}

Allow as much bone resection as need to cure the infection;

Restoration of bone continuity decreasing the distal stress shielding; bone fusion between host bone and allograft protects implant of excessive loading and reduces risk of implant breakage;

Restoration of abduction function by repair of the gluteus tendons with unresorbable sutures on the allograft. 


\section{Conclusion}

The diagnosis and treatment of prosthetic infection remain challenging. Rigorous multidisciplinary cooperation can minimize damages caused. The challenge is even greater when combined with resorption or substantial femoral bone damage. Treatment options are limited. Optimal management depends on proper analysis of the clinical features and the type of infection. The definitive diagnosis of infection is made on bacteriology, histology and microbiology testing, although routine ESR and CRP tests may guide the diagnosis. The use of massive allografts in septic femoral loosening classified as Paprosky III is attractive. Massive allografts are common indications in oncologic surgery and aseptic hip prostheses revision. There is little in the literature to support their indication in septic revisions. They restore bone stock and provide immediate hip stability. Their use remains attractive, however, there are still limiting factors, such as the necessity of a reliable tissue bank with quality allografts in sufficient number.

\section{Conflict of Interest}

None.

\section{References}

[1] Hellmann, M., Mehta, S.D., Bishai, D.M. and Zeniman, J.M. (2010) The Estimated Magnitude and Direct Hospital Cost of Prothesis Joint Infection in the United States, 1997 to 2004. The Journal of Arthroscopy, 25, 766-771. http://dx.doi.org/10.1016/j.arth.2009.05.025

[2] Del Pozo, J.L. and Patel, R. (2009) Infection Associated with Prosthetic Joints. The New England Journal of Medicine, 361, 787-794. http://dx.doi.org/10.1056/NEJMcp0905029

[3] Zimmerli, W., Trampuz, A. and Ochner, P.E. (2004) Current-Concepts-Prosthetic-Joint Infections. The New England Journal of Medicine, 351, 1645-1654. http://dx.doi.org/10.1056/NEJMra040181

[4] Marculescu, C.E., Berbari, E.F., Hanssen, A.D., et al. (2006) Outcome of Prosthetic Joint Infections Treated with Debridement and Retention of Components. Clinical Infectious Diseases, 42, 471-478. http://dx.doi.org/10.1086/499234

[5] Hseih, P.H., Shih, C.H., Lee, M.S., Yang, W.E. and Shih, H.N. (2005) Treatment of Deep Infection of the Hip Associeted with Massive Bone Loss. The Journal of Bone \& Joint Surgery, 87-B, 770-775.

[6] Santaguida, P.L., Hawker, G.A., Hudak, P.L., Glazier, R., et al. (2008) Patients Characteristics Affecting the Prognosis of Total Hip and Knee Joint Arthroplasty: A Systematic Review. Journal Canadien de Chirurgie, 51, 428-436.

[7] Yoo, J.J., Kwon, Y.S., Koo, K.H., Yoon, K.S., et al. (2009) One-Stage Cementless Revision Arthroplasty for Infected Hip Replacements. Internal Orthopaedics, 33, 1195-1201. http://dx.doi.org/10.1007/s00264-008-0640-X

[8] McKenna, P.B., O’shea, K. and Masterson, E.I. (2009) Two-Stage Revision of Infected Hip Arthroplasty Using a Shortened Post-Operative Course of Antibiotics. Archives Orthopaedic and Trauma Surgery, 129, 484-494. http://dx.doi.org/10.1007/s00402-008-0683-x

[9] Nusem, L. and Morgan, D.A.F. (2006) Structural Allografts for Bone Stock Reconstruction in Two-Stage Revision for Infected Total Hip Arthroplasty-Good Outcome in 16 of 18 Patients Followed for 5 - 14 Years. Acta Orthopaedica, 77, 92-97. http://dx.doi.org/10.1080/17453670610045740

[10] English, H., Temperley, A.J. and Dunlop, G.G. (2002) Impacting Grafting of the Femur in Two-Stage Revision for Infected Total Hip Remplacement. The Journal of Bone \& Joint Surgery, 84-B, 700-705. http://dx.doi.org/10.1302/0301-620X.84B5.12504

[11] Charrois, O., Kerboul, L., Vastel, L., Courpied, J.P. and Kerboull, M. (2000) Reconstruction fémorale par une allogreffe massive implantée dans un fémur ouvert. Revue de Chirurgie Orthopédique, 86, 801-808.

[12] Blacley, H.R.L., Davis, A.M., Hutchinson, C.R. and Gross, A.E. (2001) Proximal Femoral Allograft for Reconstruction of Bone Stock in Revision Arthroplasty of the Hip: A Nine Years Follow-Up. The Journal of Bone \& Joint Surgery, 83-A, 346-354.

[13] Alexeeff, M., Mohamed, N., Morsi, E., Garbuz, D., et al. (1996) Structural Allograft in Two Stage Revision for Failed Septic Hip Athroplasty. The Journal of Bone \& Joint Surgery, 78-B, 213-216.

[14] Ilyas, I. and Morgan, D.A.F. (2001) Massive Structural Allograft in Revision of Septic Hip Arthroplasty. Internal Orthopaedics, 24, 319-322.

[15] Matthews, P.C., Berendt, A.R., McNally, M.A. and Byren, I. (2009) Diagnosis and Management of Prosthetic Joint Infection. British Medical Journal, 338, 1378-1383. http://dx.doi.org/10.1136/bmj.b1773 
[16] Merle d’Aubigné, R. (1990) Cotation chiffrée de la fonction de la hanche. Revue de Chirurgie Orthopédique, 76, $371-374$.

[17] Bernard, L., Lübbeke, A., Stern, R., Bru, J.P., et al. (2004) Value of Preoperative Investigations in Diagnosing Prosthetic Joint Infection: Retrospective Cohort Study and Literature Review. Scandinavian Journal of Infectious Diseases, 36, 410-416. http://dx.doi.org/10.1080/00365540410015240

[18] Marculescu, C.C., Berbari, E.F., Hanssen, A.D., Steckelberg, J.M., et al. (2005) Prosthetic Joint Infection Diagnosed Postoperatively by Intraoperative Culture. Clinical Orthopaedic Related and Research, 439, 38-42. http://dx.doi.org/10.1097/01.blo.0000183091.83509.d8

[19] Jacquier, A., Champsaur, P., Vidal, V., Stein, A., et al. (2004) Evaluation des infections de prothèses totales de hanches au scanner. Journal de Radiologie, 85, 2005-2012. http://dx.doi.org/10.1016/S0221-0363(04)97773-6

[20] Piriou, P., De Loynes, B., Garreau De Loubresse, C. and Judet, T. (2003) Intérêt de la scintigraphie couplée galliumtechnétium afin d'optimiser le délai de réimplantation prothétique en deux temps des infections sur arthroplastie de hanche. Revue de Chirurgie Orthopédique, 89, 287-296.

[21] Haddad, F.S., Garbuz, D.S., Masri, B.S. and Buncan, C.P. (2000) Structural Proximal Femoral Allografts for Failed Total Hip Replacements. The Journal of Bone \& Joint Surgery, 82-B, 830-836. http://dx.doi.org/10.1302/0301-620X.82B6.10485

[22] Cornu, O., Bavadekar, A. and Godts, B. (2003) Impacting Grafting with Freeze-Dried Irradiated Bone. Part I: Femoral Implant Stability. Acta Orthopaedica, 74, 547-552. http://dx.doi.org/10.1080/00016470310017938

[23] Malhotra, R., Dua, A., Kiran, E.K. and Bhan, S. (2008) Femoral Revision Using Long Hydroxyapatite-Coated Interlocking Stem. Archives of Orthopaedic and Trauma Surgery, 128, 355-362. http://dx.doi.org/10.1007/s00402-007-0334-7

[24] Phillips, J.E., Crane, T.P., Elliott, T.S. and Grimer, R.J. (2006) The Incidence of Deep Prosthesis Infection in a Specialist Orthopaedic Hospital: A 15-Years Survery. The Journal of Bone \& Joint Surgery, 88-B, 943-948. http://dx.doi.org/10.1302/0301-620X.88B7.17150

[25] Delloye, C., Suratwala, S., Cornu, O. and Lee, F. (2004) Treatment of Allograft Nonunions with Recombinant Human Bone Morphogenetic Proteins (rh BMP). Acta Orthopaedica Belgica, 70, 591-597.

[26] Hamer, A.J., Strachan, J.R., Black, M.M., Ibbotson, C.J., et al. (1996) Biomechanical Properties of Cortical Allograft Bone Using a New Methode of Bone Strength Measurement. A Comparison of Fresh, Fresh-Frozen and Irradied Bone. The Journal of Bone \& Joint Surgery, 78-B, 363-368.

[27] Halliday, B.R., English, H.W., Timperley, A.J. and Ling, R.S.M. (2003) Femoral Impaction Grafting with Cement in Revision Total Replacement. Evolution of the Technique and Results. The Journal of Bone \& Joint Surgery, 85-B, 809-817.

[28] Kwee, T.C., Kwee, R.M. and Alavi, A. (2008) FDG-PET for Diagnosing Prosthetic Joint Infection: Systematic Review and Metaanalysis. European Journal Nuclear Medicine and Molecular Imaging, 35, 2122-2132. http://dx.doi.org/10.1007/s00259-008-0887-X

[29] Vandercam, B., Jeumont, S., Cornu, O., Yombi, J., et al. (2008) Amplification-Based DNA Analysis in the Diagnosis of Prosthetic Joint Infection. Journal of Molecular Diagnostics, 10, 537-543. http://dx.doi.org/10.2353/jmoldx.2008.070137

[30] Alboltins, C.A., Page, M.A., Buising, K.L., Jenney, A.W., et al. (2007) Treatment of Staphylococcal Prosthesis Joint Infection with Debridement, Prosthesis Retention and Oral Rifampicin and Fusidic Acid. Clinical Microbiology and Infection, 13, 586-691. http://dx.doi.org/10.1111/j.1469-0691.2007.01691.x

[31] Berbari, E.F., Osmon, D.R., Duffy, M.C.T., Harmssen, R.N.W., et al. (2006) Outcome of Prosthetic Joint Infection in Patients with Rheumatoid Arthritis: The Impact of Medical and Surgical Therapy in 200 Episodes. Clinical Infectious Diseases, 42, 216-223. http://dx.doi.org/10.1086/498507

[32] De Thomasson, E., Guingand, O. and Mazel, C. (2000) Modified Exeter Technique for the Reconstruction of Femoral Bone Loss in Revision Total Hip Arthroplasty. Does Prosthesis Stability Affect Remodeling of the Graft? European Journal of Orthopaedic Surgery \& Traumatology, 10, 35-42. http://dx.doi.org/10.1007/BF02803171

[33] Van Kleunen, J.P., Anbari, K.K., Vu, D. and Garino, J.P. (2006) Impaction Allografting for Massive Femoral Defects in Revision Hip Arthroplasty Using Collared Textured Stems. The Journal of Arthroplasty, 21, 362-371. http://dx.doi.org/10.1016/j.arth.2005.04.041

[34] Philippot, R., Dalangle, F., Verdot, F.X., Farizon, F. and Fessy, M.H. (2009) Femoral Deficiency Reconstruction Using a Hydroxyatatite-Coated Locked Modular Stem. A Series of 43 Total Hip Revisions. Orthopaedics \& Traumatology: Surgery \& Research, 95, 119-126. http://dx.doi.org/10.1016/j.otsr.2008.09.009

[35] Markel, M., Gottsauner-Wolf, F., Rock, M.G., Frassica, F.J. and Choa, E.Y.S. (1993) Mechanical Characteristics of Proximal Femoral Reconstruction after 50\% Resection. Journal of Orthopaedic Research, 11, 339-349. http://dx.doi.org/10.1002/jor.1100110305 